Pacific

Journal of

Mathematics

\title{
HIGHER EXCHANGE RELATIONS
}

ShamindRa Kumar GHOSH 


\title{
HIGHER EXCHANGE RELATIONS
}

\author{
Shamindra Kumar Ghosh
}

In this paper, we extend Landau's notion of 'exchange relations' so as to make sense for arbitrary planar algebras, which need not necessarily be generated by its '2-boxes'. We show, as in Landau's case, that these 'higher exchange relation planar algebras' are necessarily 'finite dimensional', and that examples of such planar algebras are given by all (even possibly reducible) depth two subfactors, as well as planar algebras associated to subfactors with principal graphs $E_{6}$ and $E_{8}$.

\section{Introduction}

In [Jon1], V.F.R. Jones introduced the notion of index for Type $\mathrm{II}_{1}$ subfactors and also examined the canonical tower,

$$
N \subset M \subset M_{1} \subset M_{2} \subset \cdots
$$

of basic construction of $N \subset M$. For a finite index inclusion of Type $\mathrm{II}_{1}$ factors, $N \subset M$, the grid of finite dimensional algebras of relative commutants,

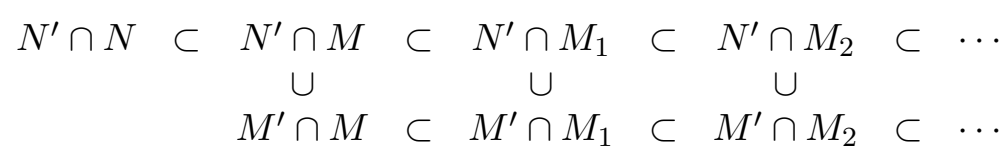

known as the standard invariant, became an important invariant for $N \subset M$ (see [GHJ], [JS], [Pop1], [Pop2]).

Popa ([Pop2]) studied the question of which families $\left\{A_{i j}:-1 \leq i \leq\right.$ $j<\infty\}$ of finite-dimensional $C^{*}$-algebras could arise as the tower of relative commutants of an extremal finite-index subfactor - i.e., when does there exist such a subfactor $M_{-1} \subset M_{0}$ such that $A_{i j}=M_{i}^{\prime} \cap M_{j}$; and he obtained a beautiful algebraic axiomatisation of such families, which he called $\lambda$ lattices. Subsequently, Jones used this characterisation of $\lambda$-lattices to obtain an algebraic and geometric reformulation of the standard invariant, which he called Planar Algebras (see [Jon2]).

In [Jon2], a construction of planar algebras by generators and relations is described. In [Lan], a condition called 'the exchange relation' is obtained, which guarantees that a planar algebra 'generated by finitely many of its 2boxes' satisfies the crucial requirement of finite dimensionality. In this paper we extend and generalise the 'exchange relation' condition in [Lan]; we relax 
the '2-box' requirement of the generating set in [Lan] and define a 'higher exchange relation'. Our definition does not demand the planar algebra to be irreducible (as in [Lan]); but puts the restriction that the $A T L_{1}$-submodule (in the sense of [Jon3]) generated by the generators of the planar algebra is closed under multiplication. We show that such 'higher exchange relation planar algebras' are indeed finite dimensional (see Theorem 6). The ideas of the proof of Lemma 5 and Theorem 6 have a similar flavour to that of Theorem 1 in [Lan], namely, the 'internal face' consideration and the use of Euler characteristic formula. However, the computations are more involved due to the fact that the planar algebras are generated by ' $k$-boxes' instead of '2-boxes'. Essential to these computations are the notions that we have termed ' $i$-capped $k$-boxes' and 'proper internal face' (which latter notion was introduced instead of the 'internal face' of [Lan], where only the irreducible case is treated). Proposition 8 shows that any depth two subfactor corresponds to a higher exchange relation planar algebra; this generalizes Theorem 4 in [Lan], where only irreducible depth two subfactors were considered. (It should be noted that an exchange relation planar algebra in the sense of $[\mathbf{L a n}]$ is also a higher exchange relation planar algebra.) We conclude by showing that the planar algebras associated to the $E_{6}$ and $E_{8}$ subfactors are higher exchange relation planar algebras.

\section{Planar algebra.}

We recall the notion of planar algebra in the form of generators and relations as described in [Jon2]. We first give some definitons.

For $k \geq 0$, by a standard $k$-box, we will mean a rectangle — or sometimes a circle - with $2 k$ points which are numbered in a clockwise fashion.

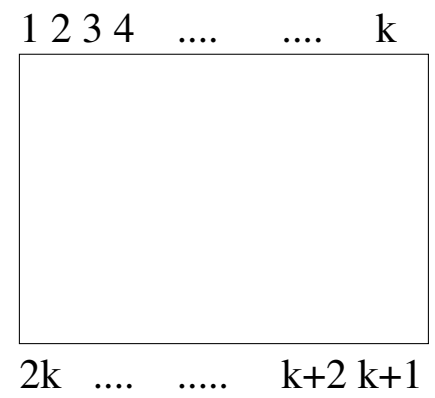

Figure 1.

We also need a labelling set $L=\coprod_{k=0}^{\infty} L_{k}$ - where $\coprod$ denotes disjoint union. A standard $k$-box will be said to be $L$-labelled if it has been assigned an element of $L_{k}$. 
Definition 1. An $L$-labelled $k$-tangle, for $k \geq 1$ consists of:

(a) A standard $k$-box which forms the external boundary,

(b) finitely many (may be zero) $L$-labelled standard $l$-boxes inside the external standard $k$-box, for $l \geq 1$,

(c) oriented planar strings, some of which may constitute loops, the others connecting two boxes in such a way that each of the marked points on the boxes is attached to exactly one string which ends at some other marked point, and the orientation of the strings satisfies the following rules:

(i) A string attached to an odd point of the external box or an even point of an internal box is oriented towards the point;

(ii) a string attached to an even point of the external box or an odd point of an internal box is oriented away from the point.

(We are given a checkerboard shading of the connected components of the complement of the strings and internal boxes inside a $k$-tangle, whereby, as one moves along the orientation of any string, the region to the right (resp., left) is shaded (resp., unshaded).)

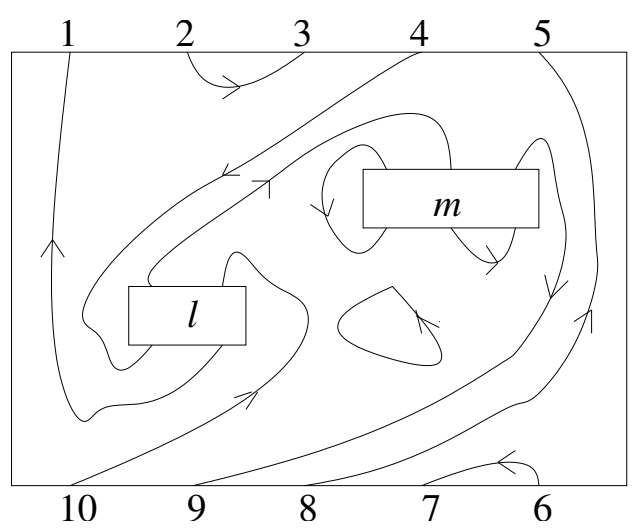

Figure 2.

We consider tangles up to planar isotopy. So, an $L$-labelled $k$-tangle is an equivalence class of pictures defined above under planar isotopy of the strings. Figure 2 shows an example of $L$-labelled 5 -tangle with two internal boxes, and $l \in L_{2}, m \in L_{3}$. After shading, the tangle looks like Figure 3.

In Figure 3, the *'s, present in the white region near the boundary of the external disc and the two internal discs, indicate the white region adjacent to the first point in each disc. Similarly, in any tangle, instead of numbering each point of the external or internal boxes, we can just put a $*$ near the boundary of the box in the white region adjacent to the first point. 


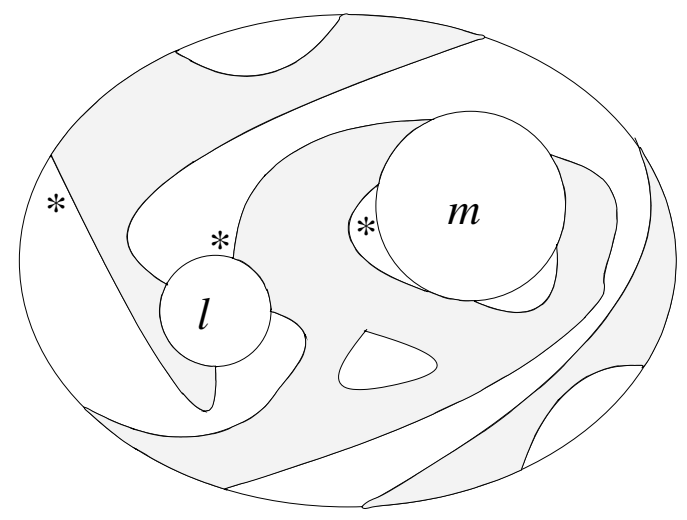

Figure 3.

On the other hand, let us define an $L$-labelled 0-tangle by Conditions (a), (b), (c) of the above definiton for $k=0$, as well as the requirement that the connected component adjacent to the external boundary is unshaded.

Let $\mathcal{T}_{k}(L)$ denote the set of all $L$-labelled $k$-tangles and let $\mathcal{P}_{k}(L)$ be the vector space with $\mathcal{T}_{k}(L)$ as a basis. Then $\mathcal{P}_{k}(L)$ has a natural algebra structure, where basis elements - i.e., tangles - are multiplied by juxtaposition (as with braids). From the braid analogy, it is no surprise that (a) the identity element of $\mathcal{P}_{k}(L)$ is given by the identity tangle where there are $k$ strings going 'straight down', and that (b) $\mathcal{P}_{k}(L)$ may be identified with the subalgebra of $\mathcal{P}_{k+1}(L)$ in such a way that an element of $\mathcal{T}_{k}(L)$ is thought of as an element of $\mathcal{T}_{k+1}(L)$ whose 'right-most strand comes straight down'.
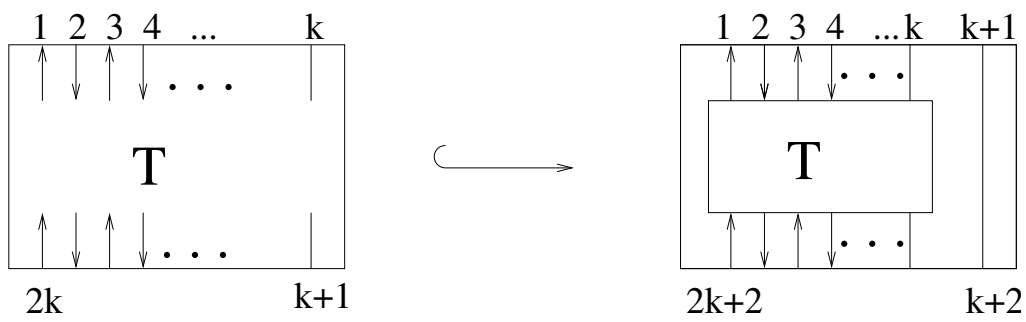

Figure 4 .

So we have a chain of unital algebras, namely,

$$
1 \in \mathcal{P}_{0}(L) \subset \mathcal{P}_{1}(L) \subset \mathcal{P}_{2}(L) \subset \cdots \bigcup_{k \geq 0} \mathcal{P}_{k}(L) .
$$

$\bigcup_{k>0} \mathcal{P}_{k}(L)$ is called the Universal Planar Algebra on $L$, and is denoted by $\mathcal{P}(\bar{L})$. 
Next, we recall the notion of annular action. By an $L$-labelled $j$ - $k$-annular tangle, we shall mean a $k$-tangle with a distinguished unlabelled internal $j$ box , all other internal boxes being $L$-labelled. Let $\mathcal{A}_{j, k}(L)$ denote the set of all $L$-labelled $j$ - $k$-annular tangles. Each $A \in \mathcal{A}_{j, k}(L)$ defines a natural linear map $A: \mathcal{P}_{j}(L) \rightarrow \mathcal{P}_{k}(L)$ thus: If $T \in \mathcal{T}_{j}(L)$, then $A(T)$ is defined as the $k$-tangle obtained by gluing the external boundary of $T$ with the boundary of the distinguished $j$-box of $A$ in such a way that the marked points of both these boundaries 'match' under the gluing; the action of $A$ is then extended linearly over $\mathcal{P}_{j}(L)$.

It should be clear that this defines an 'action' in the sense that if also $B \in \mathcal{A}_{k, m}(L)$, then, the result of 'gluing' $A$ into the distinguished internal $k$-box of $B$ - taking care to glue pairs of identically marked points — yields an element $B \circ A \in \mathcal{A}_{j, m}(L)$; and

$$
(B \circ A)(T)=B(A(T)) \text {. }
$$

We are finally ready to define $P\langle L, \mathcal{R}\rangle$, the general planar algebra with generators $L$ and relations $\mathcal{R}$.

First fix a subset $\mathcal{R} \subset \mathcal{P}(L)$ and define $\mathcal{I}_{k}(\mathcal{R})=\operatorname{span}\{A(X): A \in$ $\left.\mathcal{A}_{j, k}(L), X \in \mathcal{R} \cap \mathcal{P}_{j}(L), j \geq 0\right\}$. It turns out (see [Jon2]) that $\mathcal{I}_{k}(\mathcal{R})$ is an ideal in $\mathcal{P}_{k}(L)$, and that $\mathcal{I}_{k+1}(\mathcal{R}) \cap \mathcal{P}_{k}(L)=\mathcal{I}_{k}(\mathcal{R})$. Therefore, $\mathcal{I}(\mathcal{R})=$ $\bigcup_{k \geq 0} \mathcal{I}_{k}(\mathcal{R})$ is an ideal in $\mathcal{P}(L)$.

Consider the quotient map $\Phi_{\mathcal{R}}: \mathcal{P}(L) \rightarrow \frac{\mathcal{P}(L)}{\mathcal{I}(\mathcal{R})}=P\langle L, \mathcal{R}\rangle$. Define $P_{k}\langle L, \mathcal{R}\rangle=\frac{\mathcal{P}_{k}(L)}{\mathcal{I}_{k}(\mathcal{R})}=\Phi_{\mathcal{R}}\left(\mathcal{P}_{k}(L)\right)$. Thus we have another chain of unital algebras:

$$
P_{0}\langle L, \mathcal{R}\rangle \subset P_{1}\langle L, \mathcal{R}\rangle \subset P_{2}\langle L, \mathcal{R}\rangle \subset \cdots \bigcup_{k \geq 0} P_{k}\langle L, \mathcal{R}\rangle=P\langle L, \mathcal{R}\rangle .
$$

We say $P\langle L, \mathcal{R}\rangle$ is a planar algebra ${ }^{1}$ if it satisfies the following two conditions:

(i)

$$
\operatorname{dim}\left(P_{0}\langle L, \mathcal{R}\rangle\right)=1=\operatorname{dim}\left(P_{1,1}\langle L, \mathcal{R}\rangle\right)
$$

(ii) both

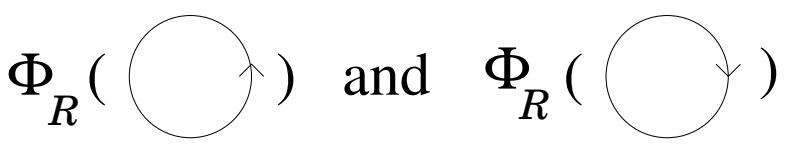

are nonzero.

\footnotetext{
${ }^{1}$ It must be remarked that in order to make contact with subfactors - like Jones it is necessary to also require that what we have called a planar algebra should satisfy further conditions so as to become 'finite-dimensional, spherical and $C^{*}$-planar algebras'.
} 
In Condition (i) of the above definition, we have, following [Jon2], used the notation $P_{1,1}\langle L, \mathcal{R}\rangle=\Phi_{\mathcal{R}}\left(\mathcal{P}_{1,1}(L)\right)$ and

$$
\mathcal{P}_{1,1}(L)=\operatorname{span}\left\{\begin{array}{l|l}
T \in \mathcal{P}_{1}(L) & \begin{array}{l}
\text { the two points of the boundary } \\
\text { of } T \text { are connected by a string }
\end{array}
\end{array}\right\} .
$$

The map $\Phi_{\mathcal{R}}$ is said to present the planar algebra $P\langle L, \mathcal{R}\rangle$ on the labelling set $L$ with relations $\mathcal{R}$.

\section{Higher exchange relation planar algebra.}

In this section, we will define what we mean by a 'higher exchange relation planar algebra' and we will show that any finitely generated planar algebra which satisfies this condition is 'locally finite-dimensional'. (This will generalise the 'exchange relation' condition of [Lan] which, however, makes sense only for planar algebras generated by finitely many 2-boxes.)

Begin by noting that an 'annular $j$ - $k$-tangle on the empty set' (i.e., an element of $\left.\mathcal{A}_{j, k}(\phi)\right)$ is nothing but a $k$-tangle with no internal box other than the distinguished $j$-box. Now if $1 \leq i \leq k$, define an $i$-capped $k$-box to be a $(k-i)$-box obtained by applying any annular tangle in $\mathcal{A}_{k-i, k}(\phi)$ on a $k$-box; this has the effect of putting $i$ caps on the $k$-box. Note that there will be exactly $2(k-i)$ many free strings coming out of an $i$-capped $k$-box. We shall write $l^{(i)}$ to denote an $i$-capped $k$-box whose internal $k$-box is labelled by $l$.

Figure 5 gives two examples of 2-capped 3-boxes.
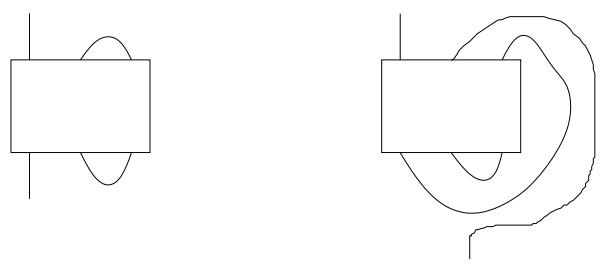

\section{Figure 5.}

Remark 2. Before proceeding to the next (and crucial, for us) definition of a 'higher exchange relation', which might be a little forbidding at first glance, it will help if we digress briefly with a comparison of Landau's original definition of an 'exchange relation' with our Definition 3.

(a) He works with the case where $P\langle L, \mathcal{R}\rangle$ is generted by its 2-boxes i.e., $L=L_{2}$ and $L_{k}=\phi$ for $k \neq 2$ - where $\phi$ denote the empty set, and where $L$ is a finite set. We work with the general finitely presented case. Without loss of generality, we may assume that there exists a $k$ such that $L_{k^{\prime}}=\phi$ if $k^{\prime} \neq k$ since if $l^{\prime} \in L_{j}$ and $j \leq k$, we may take the picture in Figure 6 as a new label, $l \in L_{k}$. 

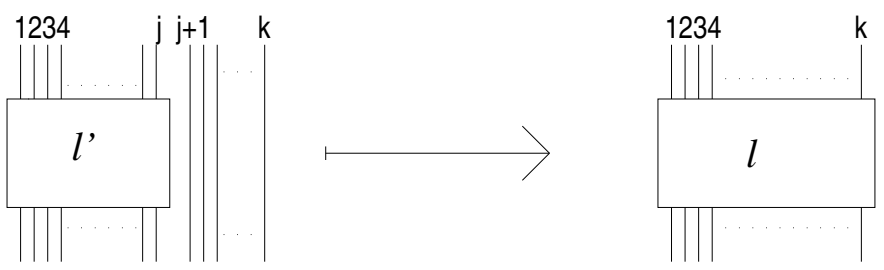

Figure 6.

(b) He needs (and implicitly uses, although he does not explicitly include this in his definition) the fact that in his planar algebra, 'contractible loops can be replaced by scalars'. We explicitly assume this as Condition (o) of Definition 3.

(c) He works with the case of 'irreducible subfactors', which amounts, in our case, to working with the case where

$$
P_{0}\langle L, \mathcal{R}\rangle=P_{1}\langle L, \mathcal{R}\rangle=\mathbb{C}
$$

Our Conditions (i) and (ii) of Definition 3 are appropriate generalisations of these two requirements. When $k=2$, our conditions are equivalent to demanding that $P_{0}\langle L, \mathcal{R}\rangle=\mathbb{C}$ and that $P_{1}\langle L, \mathcal{R}\rangle$ is finitedimensional.

(d) The really important condition in Landau's definition is the last condition, the one which motivated his terminology (on the basis of the 'group example'). This condition stems from the fact that there are essentially three different kinds of 3-tangles which have two internal 2-boxes connected by one string; and the condition requires that any labelled tangle of one of these kinds is expressible as linear combinations of labelled tangles of the other 2-kinds. The needed generalisation of this condition requires the use of the 'rotations' which play an all-important role in Jones' theory of planar algebras. (Recall that for each $j \geq 1$, the rotation is the annular $j$ - $j$ tangle $\rho_{j} \in \mathcal{A}_{j, j}(\phi)$ where the point marked 1 on the internal disc is connected to the point marked 3 on the external disc.) According to [Jon2], the map $T \mapsto \rho_{j}(T)$ defines a bijective self-map of $P_{j}\langle L, \mathcal{R}\rangle$ which, in the subfactor case, preserves the 'inner-product induced by the trace'.

Definition 3. $P\langle L, \mathcal{R}\rangle$ is said to be a higher exchange relation planar algebra if $\mathcal{R}$ contains the following elements:

(o) If $\gamma$ is a contractible loop, then

$$
(\gamma-\delta 1) \in \mathcal{R} \text { for some scalar } \delta
$$


(i) For any $k$-capped labelled $k$-box, $l^{(k)}$ (with $l \in L=L_{k}$ ), there exists a constant $C\left(l^{(k)}\right)$ such that

$$
l^{(k)}-C\left(l^{(k)}\right) 1 \in \mathcal{R} .
$$

(ii) For any three $(k-1)$-capped labelled $k$-boxes, $l^{(k-1)}, m^{(k-1)}$ and $n^{(k-1)}$, there exists constants $C_{n^{(k-1)}}^{l^{(k-1)}, m^{(k-1)}}$ such that

$$
\left(l^{(k-1)} \cdot m^{(k-1)}-\sum_{n^{(k-1)}} C_{n^{(k-1)}}^{l^{(k-1)}, m^{(k-1)}} n^{(k-1)}\right) \in \mathcal{R} .
$$

(This relation says that $\operatorname{span}\left\{\Phi_{\mathcal{R}}\left(l^{(k-1)}\right): l \in L\right\}$ is closed under multiplication in $P\langle L, \mathcal{R}\rangle$.)

(iii) Let $A$ denote any $(2 k-1)$-tangle with exactly two internal $k$-boxes, both unlabelled, which are connected by exactly one string, in which all other strings have exactly one end point on the external disc, but whose three *'s (one for the external and two for the internal boxes) can be in arbitrary places (modulo the shading requirement).

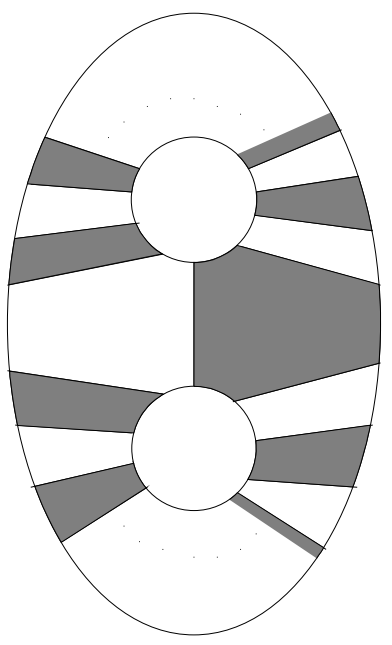

Figure 7.

For each $A$ above and any $l, m \in L=L_{k}$ we demand that $A(l, m)$ can be written as the sum of two terms: The first term is a suitable linear combination of $L$-labelled $(2 k-1)$-tangles obtained by applying nontrivial rotations on the external boundary of $A$ and arbitrary rotations on the two internal boxes which can assume any lables from $L$, the second term is a suitable linear combination of $(2 k-1)$ tangles with at most one labelled $k$-box. More precisely, if we denote by $\Lambda$, the set of all $(2 k-1)$ tangles which have at most one $L$-labelled internal 
box, then we require the existence of constants $C_{x, y, i, p, q}^{A, l, m}$ and $C_{T}^{(A, l, m)}$ such that

$$
A(l, m)-\left(\sum_{\substack{x, y \in L, 1 \leq p, q \leq k \\ 1 \leq i \leq(2 k-2)}} C_{(x, y, i, p, q)}^{(A, l, m)} \rho_{2 k-1}^{i}\left(A\left(\rho_{k}^{p}(x), \rho_{k}^{q}(y)\right)\right)+\sum_{T \in \Lambda} C_{T}^{(A, l, m)} T\right) \in \mathcal{R} .
$$

\section{Remark 4.}

(a) Note that in Condition (iii), the symbol $i$ runs from 1 to $(2 k-2)$ which means that we consider only nontrivial rotations of the external boundary, whereas we also allow the trivial rotation (i.e., the identity map) of the internal discs.

(b) In Condition (iii), instead of making $*$ for the external boundary of $A$ arbitrary, we can just demand the fact for a fixed location of the $*$ of the external boundary. This is because the relation for other positions of $*$ can be deduced by applying nontrivial rotations to the external boundary of $A$.

The interior of each tangle is partitioned by the strings and the internal boxes; the connected components will be called faces. Each face which does not touch the external boundary of the tangle will be called an internal face and the size of an internal face is defined as the number of internal boxes touched by the face. An internal face will be said to be a proper internal face, if its size is strictly greater than 1 .

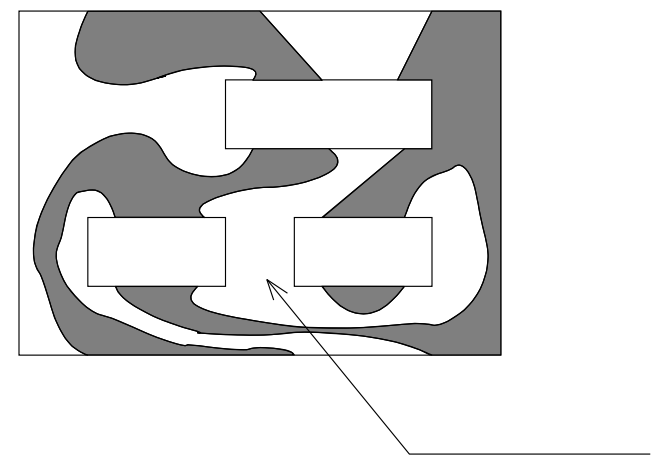

proper internal face

\section{of size 3}

Figure 8.

Lemma 5. If $P(=P\langle L, \mathcal{R}\rangle)$ is a higher exchange relation planar algebra, and if $T \in \mathcal{T}_{n}(L), n \geq 0$, then $\Phi_{\mathcal{R}}(T)$ can be written as a linear combination of terms of the form $\Phi_{\mathcal{R}}\left(T^{\prime}\right)$ where $T^{\prime} \in \mathcal{T}_{n}(L)$ and does not contain any proper internal face. 
Proof. Suppose not. Let

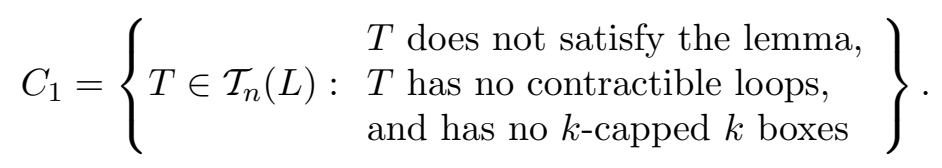

Then $C_{1} \neq \phi$, by assumption and by relations (o), (i) of Definition 3 . Choose $T_{1} \in C_{1}$ such that number - call it $n_{1}$ - of internal boxes in $T_{1}$ is minimal among all elements $T \in C_{1}$.

Let $C_{2}=\left\{T \in C_{1}\right.$ : number of internal boxes in $\left.T=n_{1}\right\} . C_{2} \neq \phi$ since it contains $T_{1}$. Choose $T_{2} \in C_{2}$ such that the size of its smallest proper internal face - call it $n_{2}$ - is minimal among all elements $T \in C_{2}$. (This makes sense because each element of $C_{1}$ has at least one proper internal face.) By definition of proper internal face, $n_{2} \geq 2$. Observe that inside $T_{2}$, we can find a picture of Type $A\left(l, l^{\prime}\right)$ (as in Definition 3(iii)), so that the string connecting the two discs is on the boundary of a proper internal face of $T_{2}$ of size $n_{2}$. (See Figure 9.)

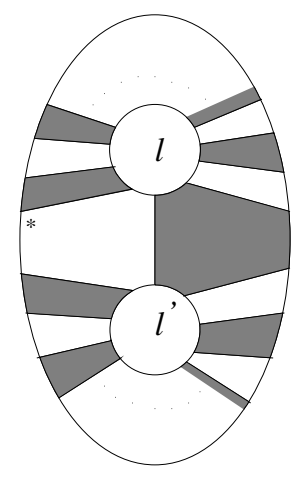

\section{Figure 9.}

Using relation (iii), $A\left(l, l^{\prime}\right)$ can be replaced by a linear combination of certain pictures and thereby $\Phi_{\mathcal{R}}\left(T_{2}\right)$ can be written as a linear combination of $\Phi_{\mathcal{R}}\left(T^{\prime}\right)$ s where $T^{\prime} \in \mathcal{P}_{n}(L)$. The crucial point is that while we write $\Phi_{\mathcal{R}}\left(T_{2}\right)$ as a linear combination of $\Phi_{\mathcal{R}}\left(T^{\prime}\right)$ s via expressing $A\left(l, l^{\prime}\right)$ as a linear combination of certain pictures, each $T^{\prime}$ is seen to necessarily satisfy one of the following two conditions:

(i) The number of internal boxes of $T^{\prime}$ is strictly less than $n_{1}$; or

(ii) $T^{\prime}$ has exactly $n_{1}$ internal boxes and the minimal size of its proper internal faces is strictly less than $n_{2}$.

Finally observe that the assertion of the lemma is indeed satisfied by $T^{\prime}$ 's of either of the above types, by the definitions of $n_{1}$ and $n_{2}$. Thus, the assertion of the lemma is satisfied by all $T^{\prime}$ 's which appear in the linear 
combination yielding $\Phi_{\mathcal{R}}\left(T_{2}\right)$. Hence, $T_{2}$ must also satisfy the claim. This is a contradiction and completes the proof of Lemma 5 .

Theorem 6. If $P(=P\langle L, \mathcal{R}\rangle)$ is a higher exchange relation planar algebra, then $P$ is a locally finite-dimensional planar algebra - i.e., $P_{k}$ is finite dimensional for all $k$.

Proof. We first prove that $P_{0}$ has dimension 1. It is enough, by Lemma 5 to prove that $\Phi_{\mathcal{R}}(T) \in \mathbb{C}$. 1 for $T \in \mathcal{T}_{0}(L)$ such that $T$ does not have any proper internal face. Consider such a $T$. Note that $T$ cannot have two internal boxes connected by a string between them. Otherwise, there would always exist an internal face of size $\geq 2$, because the boundary of $T$ is not connected to any string. Thus all internal $(k$-)boxes in $T$ will be $k$-capped. So, $T$ will consist of $k$-capped internal boxes and contractible loops; then, using the relations, (o) and (i), we find that $\Phi_{\mathcal{R}}(T) \in \mathbb{C} .1$.

Similarly, we can prove that $P_{1,1}=\mathbb{C} .1$ Thus, $P$ is a planar algebra. It remains to show that $\operatorname{dim}\left(P_{n}\right)<\infty$.

Suppose $T \in \mathcal{P}_{n}(L)$ and $T$ does not contain any proper internal face, contractible loop or $k$-capped box. Let $b^{T}$ be the number of internal boxes in $T$ and $b_{i}^{T}$ be the number of $i$-capped boxes in $T$. Clearly, $b^{T}=\sum_{i=0}^{k-1} b_{i}^{T}$.

We construct a graph (embedded in the 2-sphere) from $T$ thus: Embed the tangle $T$ in the 2-sphere (thought of as the one-point compactification of the plane); join the point at $\infty$ on the sphere to each of the boundary points of the tangle $T$; and shrink each of the $i$-capped boxes along with their $i$ caps to points.

Let $V, E$ and $F$ be the number of vertices, edges and faces of this graph. Then,

$$
\begin{aligned}
V & =2 n+b_{T}+1 \\
E & =\frac{2 \cdot(2 n)+2 n+\sum_{i=0}^{k-1} 2(k-i) b_{i}^{T}}{2} \\
& =3 n+\sum_{i=1}^{k} i b_{k-i}^{T} \\
F & \leq 2 n \text { since } T \text { does not have any proper internal face. }
\end{aligned}
$$

Since the Euler characteristic of $S^{2}$ is 2, we find that

$$
\begin{aligned}
V & -E+F=2 \\
& \Rightarrow \quad(E-V)=(F-2) \\
& \Rightarrow \quad(E-V) \leq(2 n-2) \\
& \Rightarrow(n-1)+\sum_{i=1}^{k}(i-1) b_{k-i}^{T} \leq(2 n-2) \\
& \Rightarrow \sum_{i=2}^{k}(i-1) b_{k-i}^{T} \leq(n-1)
\end{aligned}
$$




$$
\begin{gathered}
\Rightarrow \sum_{i=2}^{k} b_{k-i}^{T} \leq(n-1) \\
\Rightarrow \quad\left(b^{T}-b_{k-1}^{T}\right)=\left(\sum_{i=1}^{k} b_{k-i}^{T}\right)-b_{k-1}^{T} \leq(n-1) \\
\text { Let } D_{1}=\left\{\begin{array}{l}
T \text { has no contractible loops, } \\
T \in \mathcal{T}_{n}(L): \begin{array}{l}
\text { no } k \text {-capped box } \\
\text { no }(k-1) \text {-capped box } \\
\text { and }\left(b^{T}-b_{k-1}^{T}\right) \leq(n-1)
\end{array}
\end{array}\right\} .
\end{gathered}
$$

Clearly, $\left|D_{1}\right|<\infty$, since the labelling set is finite and since there exist only finitely many $n$-tangles with a fixed number of boxes. For $T \in D_{1}$, let us write $T^{a}$ for the result obtained by inserting exactly one $(k-1)$-capped labelled $k$-box on each of some (maybe all) of the strings of $T$. (There may be several $T^{a}$ 's for each $T \in D_{1}$, but the number of such $T^{a}$ is finite, because there are only a finite number of strings in $T$ and only finitely many $(k-1)$-capped labelled $k$-boxes.) Hence, the set $D_{2}=\left\{T^{a}: T \in D_{1}\right\}$ is finite.

We finish the proof of the theorem by showing that $D_{2}$ linearly spans $P_{n}$. Now $P_{n}$ is generated by the set of $\Phi_{\mathcal{R}}(T)$ 's, where $T$ ranges over those $n$ tangles which have no proper internal faces, contractible loops, or $k$-capped boxes. Take such a $T$. Replacing the $(k-1)$-capped boxes in $T$ by 'straight strings', we get an element of $D_{1}$. Thus $T$ is basically an element of $D_{1}$, on some of whose strings, one or more $(k-1)$-capped boxes have been inserted. By virtue of relation (ii), we can express $\Phi_{\mathcal{R}}(T)$ as a linear combination of $\Phi_{\mathcal{R}}\left(T^{\prime}\right)^{\prime}$ 's, where $T^{\prime} \in D_{2}$. Thus $\operatorname{dim}\left(P_{n}\right)<\infty$.

Remark 7. If we consider a higher exchange relation planar algebra with the labelling set $L=L_{2}$ and each $(k-1)$-capped labelled $k$-box is some scalar times identity, then the planar algebra is an Exchange Relation Planar Algebra in the sense of [Lan]. We will discuss some other examples of higher exchange relation planar algebra in the following section.

\section{Subfactors with depth 2 .}

Let $N \subset M$ be a finite index inclusion of $\mathrm{II}_{1}$ factors with depth 2 and $N \subset M \subset^{e_{1}} M_{1} \subset^{e_{2}} M_{2} \subset^{e_{3}} \ldots$ be the Jones tower of basic construction, where $e_{k}$ denotes the Jones projection in $M_{k}$ which implements the conditional expectation of $M_{k-1}$ onto $M_{k-2}$. Therefore, $N^{\prime} \cap M_{k-1} \subset N^{\prime} \cap M_{k} \subset^{e_{k+1}} N^{\prime} \cap$ $M_{k+1}$ is a basic construction of finite dimensional $C^{*}$-algebras for all $k \geq 1$.

It follows from [Jon2] that there is a planar algebra, $P^{N \subset M}$, associated to $N \subset M$ where $P_{k}^{N \subset M}=N^{\prime} \cap M_{k-1}$ for all $k \geq 0$. For this planar 
algebra, we may choose the generating set $L=L_{2}$ as the union of systems of matrix units of central summands of the 'multi-matrix algebra' $P_{2}^{N \subset M}$; and we may take the set of relations as $\mathcal{R}=\left\{X \in \mathcal{P}(L): \Phi^{N \subset M}(X)=0\right\}$ where $\Phi^{N \subset M}$ is a presenting map for $P^{N \subset M}$. (The reason we may make this choice of $L$ is that $P_{k}^{N \subset M} \subset P_{k+1}^{N \subset M} \subset P_{k+2}^{N \subset M}$ is a basic construction for all $k \geq 0$, so that $P_{k}^{N \subset M}$ as an algebra is generated by $P_{2}^{N \subset M}$ and $e_{1}$, $e_{2}, e_{3}, \ldots, e_{k-1}$.)

Let $\Phi_{\mathcal{R}}$ denote the quotient map from $\mathcal{P}(L)$ onto $P\langle L, \mathcal{R}\rangle$. The inclusion $L \subset P^{N \subset M}$ is seen to induce a planar algebra homomorphism $\Gamma$ from $P\langle L, \mathcal{R}\rangle$ into $P^{N \subset M}$ such that the following diagram commutes:

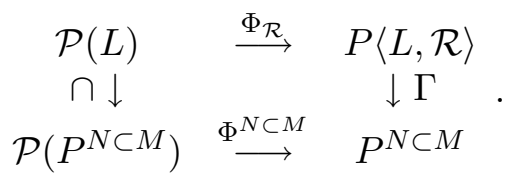

From the argument of the (parenthetical statement of the) previous paragraph, and the commutativity of the above diagram, it is seen that $\Gamma$ is surjective. Now for $X \in \mathcal{P}(L)$, if $\Gamma \circ \Phi_{\mathcal{R}}(X)=0$, then $\Phi^{N \subset M}(X)=0$ which implies $X \in \mathcal{R}$, that is, $\Phi_{\mathcal{R}}(X)=0$. Hence $\Gamma$ is $1-1$ and thereby a planar algebra isomorphism.

So we can view $P\left(=P^{N \subset M}\right)$ as a planar algebra presented on $L=L_{2}$ in the above manner, with $|L|<\infty$.

Proposition 8. Any planar algebra associated to a finite index inclusion of $\mathrm{II}_{1}$-factors with depth 2 is a higher exchange relation planar algebra.

Proof. Let $N \subset M$ be a finite index inclusion of $\mathrm{II}_{1}$-factors with depth 2. To it, we associate the planar algebra, $P\langle L, \mathcal{R}\rangle$ described above. We proceed now to systematically verify that $P\langle L, \mathcal{R}\rangle$ indeed satisfies the several requirements for being a higher exchange relation algebra.

(o) If $\gamma$ is a contractible loop, then

$$
\Phi^{N \subset M}(\gamma-\delta 1)=0 \Rightarrow(\gamma-\delta 1) \in \mathcal{R} \text { for } \delta=[M: N]^{\frac{1}{2}} .
$$

(i) A 2-capped $L$-labelled 2-box can - depending on the location of the marked point labelled 1 - be viewed as an element of $P_{0}\langle L, \mathcal{R}\rangle$ or of $P_{1,1}\langle L, \mathcal{R}\rangle$. But $P_{0}\langle L, \mathcal{R}\rangle=\mathbb{C}=P_{1,1}\langle L, \mathcal{R}\rangle$. So, for any $l \in L$, it follows that $\mathcal{R}$ contains $l^{(2)}-$ (some constant) 1 .

(ii) Since the conditional expectation of $P_{2}\langle L, \mathcal{R}\rangle=N^{\prime} \cap M_{1}$ onto $P_{1}\langle L, \mathcal{R}\rangle=$ $N^{\prime} \cap M$ is given by a scalar multiple of an appropriate 1-capping of 2-tangles in $P_{2}\langle L, \mathcal{R}\rangle$ and since $P_{2}\langle L, \mathcal{R}\rangle$ is linearly spanned by $L$-labelled 2-boxes, we see that $P_{1}\langle L, \mathcal{R}\rangle$ is linearly spanned by $\left\{\Phi_{\mathcal{R}}\left(l^{(1)}\right): l \in L\right\}$. In particular, $\operatorname{span}\left\{\Phi_{\mathcal{R}}\left(l^{(1)}\right): l \in L\right\}=P_{1}\langle L, \mathcal{R}\rangle$ is closed under multiplication. 
(iii) The depth 2 assumption says that $N^{\prime} \cap M \subset N^{\prime} \cap M_{1} \subset^{e_{2}} N^{\prime} \cap M_{2}$ is a basic construction; so $P_{3}\langle L, \mathcal{R}\rangle=P_{2}\langle L, \mathcal{R}\rangle E_{2} P_{2}\langle L, \mathcal{R}\rangle$ (where $E_{2}$ denotes the usual 'conditional expectation tangle - see [Jon2]). Again, $P_{2}\langle L, \mathcal{R}\rangle$ is the span of $L$-labelled 2-boxes (since $\mathcal{P}(L)$ is, by choice of $L$ ). Hence for $A, B \in L$, we find that

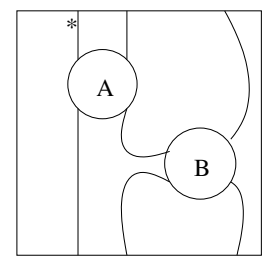

is a linear combination of

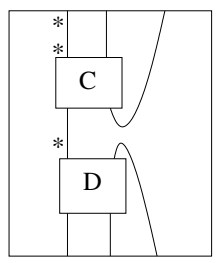

for $C, D \in L$ (* for the internal boxes with labels, $A, B$ can be arbitrary as long as the tangle makes sense). This shows that $\mathcal{R}$ satisfies Criterion (iii) in the definition of higher exchange relation planar algebras.

Thus, $P\langle L, \mathcal{R}\rangle$ is a higher exchange relation planar algebra.

\section{5. $E_{6}$ and $E_{8}$ subfactors.}

We consider the subfactors with principal graphs, $E_{6}$ and $E_{8}$, given in Figure 10 .

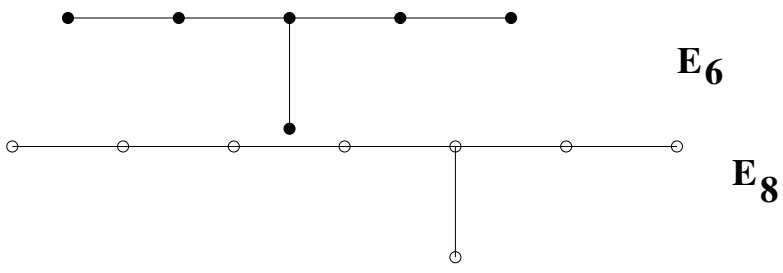

Figure 10.

Our goal is to show that the planar algebras associated to these subfactors are of the higher exchange relation type. We will be using facts stated in [Jon4]. In fact, many of the arguments here, particularly those concerned with the rotation, are from [Jon4]. 
Let $P^{6}$ (resp. $P^{8}$ ) be the planar algebra associated to the subfactor with principal graph $E_{6}\left(\right.$ resp. $\left.E_{8}\right)$. Since $E_{6}\left(\right.$ resp. $\left.E_{8}\right)$ has a unique vertex of degree 3 , which is at distance 2 (resp. 4) from $*$, we see from $[\mathbf{L a S u}]$ that $P^{6}$ (resp. $\left.P^{8}\right)$ is generated by one 3 -box, $R^{6}$ (resp. one 5 -box $R^{8}$ ) which can be taken to be any element of $P_{3}^{6} \backslash T L_{3}$ (resp. $P_{5}^{8} \backslash T L_{5}$ ). We assume $R^{6}$ (resp. $R^{8}$ ) is a unit vector orthogonal to $T L$. Since $T L$ is invariant under rotation - see $([\mathbf{J o n} 2])$ - it follows that $R^{6}$ (resp. $R^{8}$ ) is an eigenvector for $\rho_{3}$ (resp. $\rho_{5}$ ) corresponding to an eigenvalue of modulus one - in view of the 'orthogonailty' preserving nature of the rotation (see Remark 2(d), and [Jon2]).

In general, any planar algebra satisfies Conditions (o) and (i) in the definition of higher exchange relation planar algebra. So both $P^{6}$ and $P^{8}$ satisfy Conditions (o) and (i).

Next we note that our choice of $R^{6}$ (resp. $R^{8}$ ) - as an element in the orthogonal complement of the Temperley-Lieb subalgebra - implies that the result of any 1-capping of $R^{6}$ (resp. $R^{8}$ ) yields zero and consequently any 2-capping of $R^{6}$ (2-capping or 4-capping of $R^{8}$ ) is zero in $P^{6}$ (resp. $P^{8}$ ). Thus Condition (ii) is satisfied.

Now we shall see that both $P^{6}$ and $P^{8}$ satisfy Condition (iii) in the definition of a higher exchange relation planar algebra, but for different reasons.

For $P^{6}$, we have $L=L_{3}=\left\{R^{6}\right\}$. In Condition (iii) the picture, denoted by $A$, is a 5 -tangle. So we consider $P_{5}^{6}$. The dimension of $P_{5}^{6}$ can be calculated from the principal graph and turns out to be 77 .

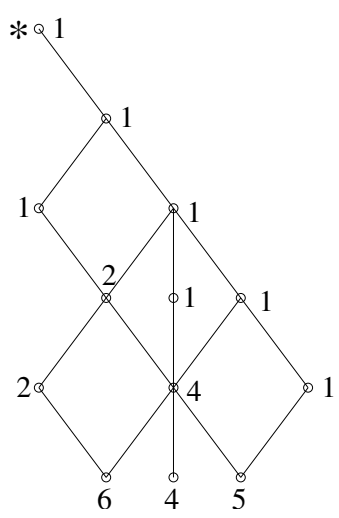

\section{Figure 11.}

Next we consider the dimension of the subspace spanned by 5 -tangles with exactly one 3 -box with label $R^{6}$. Actually there are 45 such tangles but not all of them are linearly independent. Jones [Jon4] deduces from the work [GrLe] that the dimension of the subspace spanned by these 5-tangles is 
35. This subspace lies in the orthogonal complement of the Temperley-Lieb subalgebra. We know that $\operatorname{dim}\left(T L_{5}\right)=42$. Thus we see that $\operatorname{dim}\left(P_{5}^{6}\right)=$ $77=35+42$. Therefore, $P_{5}^{6}$ is generated by 5 -tangles with atmost one 3 box. In particular, our 5-tangle $A$ of Condition (iii) is a linear combination of 5-tangles with atmost one internal 3-box. So $P^{6}$ satisfies Condition (iii), and is hence a higher exchange relation planar algebra.

For $P^{8}$, the tangle $A$ of Condition (iii) is a 9-tangle. So we consider $P_{9}^{8}$. We first look at the subspace generated by 9 -tangles with exactly one 5-box. The dimension of this subspace was found (in [Jon4], using [GrLe]) to be 2244. This subspace is also orthogonal to the Temperley-Lieb subalgebra of $P_{9}^{8}$; and we have $\operatorname{dim}\left(T L_{9}\right)=4862$. Using the principal graph, we can calculate and see that $\operatorname{dim}\left(P_{9}^{8}\right)=7107=2244+4862+1$.
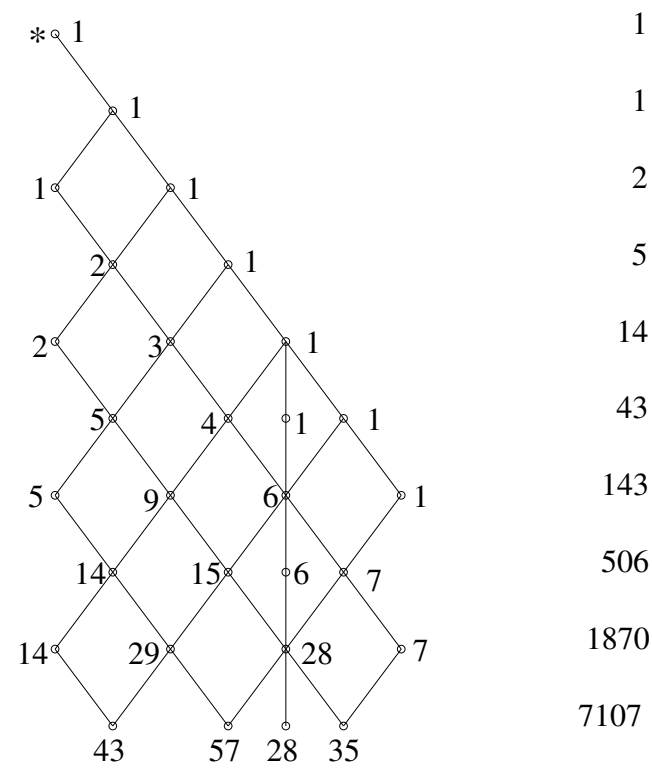

Figure 12.

Next consider the subspace - call it $W$ — which is generated by 9-tangles with at most one labelled 5-box. So we have shown that $W$ has codimension 1 in $P_{9}^{8}$. Now consider the 9-tangle in Figure 13 (where we write $R$ for $R^{8}$ ).

Let us denote the above tangle by $A_{1}$. Note that the $*$ 's for the internal boxes in $A_{1}$ are not important because $R^{8}$ forms an eigenvector for the rotation, $\rho_{9}$. If $A_{1}$ belongs to $W$, then we are through. If not, then $P_{9}^{8}$ is linearly spanned by $W \cup\left\{A_{1}\right\}$. It is enough, by Remark 4(b), to verify Condition (iii) for the 9-tangle in Figure 14, which will be denoted by $A_{2}$. 


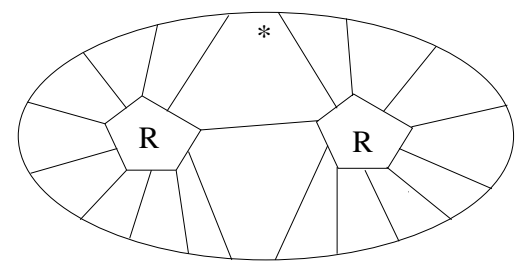

Figure 13.

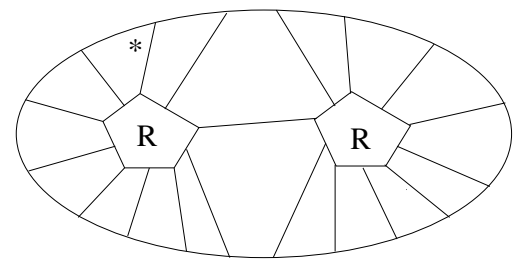

Figure 14.

Being an element of $P_{9}^{8}, A_{2}$ is a linear combination of $A_{1}$ and elements of $W$. So, $P_{9}^{8}$ satisfies Condition (iii), and is, indeed, a higher exchange relation planar algebra.

Acknowledgement. I would like to thank V.S. Sunder for valuable discussions and Zeph Landau for allowing me access to his preprint.

\section{References}

[GHJ] F. Goodman, P. de la Harpe and V.F.R. Jones, Coxeter Graphs and Towers of Algebras, Springer Verlag, MSRI publication, 1989, MR 91c:46082, Zbl 0698.46050.

[GrLe] J. Graham and G. Lehrer, The representation theory of affine Temperley-Lieb algebras, L' Enseignement Math., 44, (1998), 173-218, MR 99i:20019, Zbl 0964.20002.

[Jon1] V.F.R. Jones, Index for subfactors, Invent. Math., 72 (1983), 1-25, MR 84d:46097, Zbl 0508.46040.

[Jon2] _ Planar algebras, I, to appear in New Zealand J. Math., math.QA/9909027.

[Jon3] , The annular structure of subfactors, L' Enseignement Math., 38 (2001), 401-463, CMP 1929335.

[Jon4],$E_{6}$ is a breeze, $E_{8}$ 's a tease, Subfactors and algebraic aspects of quantum field theory conference, Fall 2000, URL: http://www.msri.org/publications/ln/ msri/2000/subfactors/jones/1/index.html.

[JS] V.F.R. Jones and V.S. Sunder, Introduction to Subfactors, Lond. Math. Soc. Lect. Notes, 234, 1997, 162 pp., MR 98h:46067, Zbl 0903.46062.

[Lan] Z.A. Landau, Exchange relation planar algebras, Geometriae Dedicata, to appear.

[LaSu] Z.A. Landau and V.S. Sunder, Planar depth and planar subalgebras, J. Funct. Anal., 195(1) (2002), 71-88, CMP 1934353. 
[Pop1] S. Popa, Classification of subfactors: The reduction to commuting squares, Invent. Math., 101 (1990), 19-43, MR 91h:46109, Zbl 0757.46054.

[Pop2] _ An axiomatization of the lattice of higher relative commutants, Invent. Math., 120 (1995), 427-445, MR 96g:46051, Zbl 0831.46069.

Received January 18, 2002 and revised July 4, 2002.

Institute of Mathematical Sciences

Chennai 600113

INDIA

E-mail address: shami@imsc.ernet.in 